\title{
CIRUGÍA PROTÉSICA AMBULATORIA EN ARTROSIS SEVERA DE CADERA Y RODI- LLA. HOSPITAL CLÍNICO METROPOLITANO DE LA FLORIDA
}

\author{
Valdés Zúñiga Claudia ${ }^{1}$, Delgado Santibañez Fernando ${ }^{1}$, Espinoza Claudia ${ }^{2}$, Paredes Orlando ${ }^{3}$ \\ Anestesiólogo Hospital de la Florida. \\ Enfermera jefa ambulatorio Hospital de la Florida. \\ Traumatólogo Hospital de la Florida.
}

Introducción: La cirugía protésica ambulatoria de cadera y rodilla, se ha desarrollado en centros específicos en Estados Unidos y Europa, sin asociar un número mayor de complicaciones y con un alto grado de satisfacción de los pacientes. A pesar de ello, la casuística y el número de publicaciones científicas es bajo.

Objetivo General: Nuestro objetivo es evaluar los resultados del programa ambulatorio en el Hospital Clínico de la Florida, que incluye tanto Artroplastias de Cadera y Rodilla.

Material y Métodos: Se realiza una evaluación prospectiva por 3 meses, de 62 pacientes de ATC y 35 pacientes de ATR con criterios ambulatorios, ASA 1 y 2. Los promedios de edad 62,9 años en ATC y 66,8 años en ATR. Los pacientes fueron seguidos por un período de 3 meses, donde se registraron a través de la ficha clínica diferentes parámetros tales como: tiempo quirúrgico, estadía postoperatoria, necesidad de transfusión sanguínea, evolución del dolor, incidencia de complicaciones quirúrgicas y sistémicas, y satisfacción del paciente. La evaluación del dolor se hizo a través de la escala visual análoga a las 4 , 24, 48 y 72 horas postoperatorio; luego en controles a la semana y al mes. Se realizaron controles de hemoglobina tomados en el postoperatorio inmediato y a las 48 horas, para ver la necesidad de transfusión. En cuanto a la satisfacción del pa- ciente y si lo recomendaría, se usa una encuesta. Resultados: El tiempo promedio de estadía hospitalaria postoperatoria para ATC fue de 5,38 horas y para ATR fue 5,4 horas. Tanto en prótesis de cadera como de rodilla no fue necesario transfusión sanguínea. Evaluación del dolor en ATC, 20\% EVA $\geq 4$ a las 24 horas, $11,3 \%$ EVA $\geq 4$ a las 48 horas Con EVA $\geq 7 ; 4,5 \%$ EVA $\geq 7$ a las 24 horas; $4.5 \%$ EVA $\geq 7$ a las 48 h. Evalución del dolor en ATR: $34 \%$ EVA $\geq 4$ a las $24 \mathrm{~h}, 31 \%$ EVA $\geq 4$ a las $48 \mathrm{~h}$. Con EVA $\geq 7: 15 \%$ EVA $\geq 7$ a las $24 \mathrm{~h} ; 18 \%$ EVA $\geq 7$ a las $48 \mathrm{~h}$. En lo que respecta a la satisfacción del paciente y si lo recomendaría a otro paciente El $100 \%$ de los pacientes tanto de cadera como rodilla, se mostraron conformes con la cirugía ambulatoria y lo recomendaría a otros pacientes.

Conclusiones: Los resultados de este trabajo demuestran que la cirugía ambulatoria de cadera y rodilla es posible de ser realizada en pacientes seleccionados en un hospital público, sin grandes recursos, con gran demanda de camas y sin grandes complicaciones quirúrgicas y anestésicas, además de contar con la aprobación de los pacientes operados. Todo esto es factible con el apoyo de un equipo multidisciplinario en el que participan traumatólogos, anestesiólogos, enfermeras, kinesiólogos y asistente social, que en conjunto permiten llevar con éxito el programa ambulatorio protésico.

Tabla 1

\begin{tabular}{|lcccc|}
\hline ATC & EVA 4 h & EVA 24 h & EVA 48 h & EVA 72 h \\
EVA $\geq 4$ & $0 \%$ & $20 \%$ & $11,3 \%$ & $2,2 \%$ \\
EVA $\geq 7$ & $0 \%$ & $4,50 \%$ & $4,5 \%$ & $4,5 \%$ \\
ATR & EVA 4 h & EVA 24 h & EVA 48 h & EVA 72 h \\
EVA $\geq 4$ & $0 \%$ & $34 \%$ & $31 \%$ & $28 \%$ \\
EVA $\geq 7$ & $0 \%$ & $15 \%$ & $18 \%$ & $12,50 \%$ \\
\hline
\end{tabular}

